\title{
ORDINARY DIFFERENTIAL SYSTEMS DESCRIBING HYSTERESIS EFFECTS AND NUMERICAL SIMULATIONS
}

\author{
EMIL MINCHEV, TAKANOBU OKAZAKI, AND NOBUYUKI KENMOCHI
}

Received 1 May 2002

We consider a general class of ordinary differential systems which describes input-output relations of hysteresis types, for instance, play or stop operators. The system consists of two first-order nonlinear ODEs and one of them includes a subdifferential operator depending on the unknowns. Our main objective of this paper is to give an existence-uniqueness result for the system as well as to give various numerical simulations of input-output relations which the system describes as typical cases.

\section{Introduction}

Consider a nonlinear system of ODEs of the following form:

$$
\begin{gathered}
a_{1}(u(t), w(t)) u^{\prime}(t)+a_{2}(u(t), w(t)) w^{\prime}(t) \\
=g(u(t), w(t)), \quad 0<t<T, \\
b_{1}(u(t), w(t)) u^{\prime}(t)+b_{2}(u(t), w(t)) w^{\prime}(t)+\partial I_{u(t)}(w(t)) \\
\ni h(u(t), w(t)), \quad 0<t<T,
\end{gathered}
$$

subject to the initial conditions

$$
u(0)=u_{0}, \quad w(0)=w_{0},
$$

where $0<T<+\infty$ and $a_{i}(\cdot, \cdot), b_{i}(\cdot, \cdot), i=1,2, g(\cdot, \cdot), h(\cdot, \cdot)$ are functions on $\mathbb{R}^{2}$ satisfying some conditions (see Section 2 ), and for each $u \in \mathbb{R}, \partial I_{u}(\cdot)$ is the subdifferential of the indicator function $I_{u}(\cdot)$ of the interval $\left[f_{*}(u), f^{*}(u)\right]$ in $\mathbb{R}$; 
namely

$$
\partial I_{u}(w)= \begin{cases}\varnothing & \text { for } w>f^{*}(u) \text { or } w<f_{*}(u), \\ {[0,+\infty)} & \text { for } w=f^{*}(u)>f_{*}(u), \\ \{0\} & \text { for } f_{*}(u)<w<f^{*}(u), \\ (-\infty, 0] & \text { for } w=f_{*}(u)<f^{*}(u), \\ (-\infty,+\infty) & \text { for } w=f_{*}(u)=f^{*}(u)\end{cases}
$$

$f_{*}(\cdot)$ and $f^{*}(\cdot)$ being nondecreasing functions such that $f_{*} \leq f^{*}$ on $\mathbb{R}$ (see Section 2 for precise conditions).

Equation (1.2) describes a lot of input-output relations $u \rightarrow w$ which are physically relevant. For instance, when $b_{1} \equiv 0$ (resp., -1 ), $b_{2} \equiv 1$, and $h \equiv 0$, the relation assigning to a function $u(t)$ the solution $w(t)$ of (1.2) is called a play (resp., stop) operator (cf. $[5,6,7])$. These operators are typical examples of hysteresis input-output relations, and are used to describe irreversible phenomena such as solid-liquid phase transition with supercooling effect and martensiteaustenite phase transition in shape memory alloys (cf. [2, 8]).

In a particular case when $a_{1} \equiv 1, a_{2} \equiv 1, b_{1} \equiv 0, b_{2} \equiv 1, g \equiv 0$, and $h \equiv 0$, the system (1.1), (1.2) was studied in detail by Visintin [7] in a very general framework; the idea for uniqueness proof is based on the so-called $L^{1}$-theory of nonlinear semigroups (cf. $[1,4]$ ), and the same idea was applied to the uniqueness proof of the Cauchy problem for (1.1), (1.2) with nonzero right-hand sides and diffusion effects in [3].

Our main objective of this paper is to give an existence-uniqueness result of the Cauchy problem for system (1.1), (1.2) under some restrictions on coefficients $a_{i}, b_{i}, i=1,2$, general enough. One of the main points in our proof is to eliminate the term $u^{\prime}$ from (1.2) to get

$$
w^{\prime}(t)+\partial I_{u(t)}(w(t)) \ni \tilde{h}(u(t), w(t)), \quad 0<t<T,
$$

with a function $\tilde{h}$ satisfying the same property as $h$, and then consider the coupling of (1.1) and (1.5). Another objective of this paper is to show by some numerical simulations that our nonlinear system covers many of physically relevant relations $u \rightarrow w$ arising in the mathematical descriptions of phase transition phenomena. In fact, a suitable choice of the set of coefficients $a_{i}, b_{i}$ and forcing terms $g, h$, can create the clockwise or anti-clockwise trend of the orbit $(u(t), w(t))$ as $t$ increases.

\section{Theoretical results}

In this section, we mention the precise assumptions on the functions $a_{i}, b_{i}, i=$ $1,2, g, h$, and theoretical results on the existence and uniqueness of a solution of (1.1), (1.2), and (1.3). 
Let $f_{*}$ and $f^{*}$ be functions on $\mathbb{R}$ such that

$f_{*}, f^{*}$ are nondecreasing and Lipschitz continuous on $\mathbb{R}$,

the derivatives $f_{*}^{\prime}:=\frac{d f_{*}}{d u}, f^{*^{\prime}}:=\frac{d f^{*}}{d u}$ are Lipschitz continuous on $\mathbb{R}$,

$$
f_{*} \leq f^{*} \text { on } \mathbb{R}
$$

$f_{*}=f^{*}$ on $\left(-\infty,-k_{0}\right] \cup\left[k_{0},+\infty\right)$ for a positive number $k_{0}$,

and put

$$
\mathscr{F}_{F}:=\left\{(u, w) \in \mathbb{R}^{2} ; f_{*}(u) \leq w \leq f^{*}(u)\right\} .
$$

For these functions $f_{*}, f^{*}$, we consider the indicator function

$$
I_{u}(w):= \begin{cases}0 & \text { for } f_{*}(u) \leq w \leq f^{*}(u) \\ +\infty & \text { otherwise }\end{cases}
$$

associated with the interval $\left[f_{*}(u), f^{*}(u)\right]$ for every $u \in \mathbb{R}$, and denote its subdifferential by $\partial I_{u}(\cdot)$ given by (1.4). Next, let $a_{i}, b_{i}, i=1,2, g, h$ be functions on $\mathscr{F}$ such that

$$
\begin{gathered}
a_{i}, b_{i}, i=1,2 \text {, are Lipschitz continuous on } \mathscr{F}, \\
a_{1} \geq c_{0} \quad \text { on } \mathscr{F}, \quad b_{2} \geq c_{0} \quad \text { on } \mathscr{F}, \\
a_{1} b_{2}-a_{2} b_{1} \geq c_{0} \quad \text { on } \mathscr{F} \text { for a positive constant } c_{0}, \\
g, h \text { are Lipschitz continuous on } \mathscr{F} .
\end{gathered}
$$

Now we give the definition of a solution of (1.1), (1.2).

Definition 2.1. A pair of (scalar) functions $\{u, w\}$ is called a solution of system (1.1), (1.2), if $u, w \in W^{1,2}(0, T)$ and they satisfy (1.1) and (1.2) a.e. on $[0, T]$; hence

$$
(u(t), w(t)) \in \mathscr{F} \quad \forall t \in[0, T] .
$$

Definition 2.2. A pair of functions $\{u, w\}$ is called a solution of (1.1), (1.2), and (1.3), if it is a solution of (1.1), (1.2) and the initial conditions $u(0)=u_{0}, w(0)=$ $w_{0}$ are satisfied.

The existence of a solution is proved under an additional condition. 
Theorem 2.3. Suppose that (2.1), (2.2), (2.3), (2.4), and (2.5) hold, and moreover suppose that there is a positive constant $\delta_{0}$ such that

$$
\begin{gathered}
a_{1}\left(u, f_{*}(u)\right)+a_{2}\left(u, f_{*}(u)\right) f_{*}^{\prime}(u) \geq \delta_{0}, \\
a_{1}\left(u, f^{*}(u)\right)+a_{2}\left(u, f^{*}(u)\right) f^{*^{\prime}}(u) \geq \delta_{0} \quad \text { for any } u \in \mathbb{R} .
\end{gathered}
$$

Then

(1) for any initial data $u_{0}, w_{0}$ satisfying $\left(u_{0}, w_{0}\right) \in \mathscr{F}$, there exists at least one solution $\{u, w\}$ of system (1.1), (1.2), and (1.3);

(2) there is a positive constant $K_{0}$, depending only on the quantities in assumptions (2.1), (2.2), (2.3), (2.4), (2.5), (2.6), and (2.7), such that

$$
\begin{gathered}
|u(t)|+|w(t)| \leq e^{K_{0}(1+T)}\left(1+\left|u_{0}\right|\right) \quad \forall t \in[0, T], \\
\left|u^{\prime}(t)\right|+\left|w^{\prime}(t)\right| \leq e^{K_{0}(1+T)}\left(1+\left|u_{0}\right|^{3}\right) \quad \text { for a.e. } t \in[0, T],
\end{gathered}
$$

for any solution $\{u, w\}$ of (1.1), (1.2), and (1.3) with initial data $u_{0}, w_{0}$.

Our second result is concerned with the uniqueness of a solution of (1.1), (1.2), and (1.3).

Theorem 2.4. Suppose that (2.1), (2.2), (2.3), (2.4), and (2.5) hold as well as

$$
a_{2} \geq \mathcal{c}_{0} \quad \text { on } \mathscr{F}
$$

where $c_{0}$ is the same positive constant as in (2.4). Then system (1.1), (1.2), and (1.3) admits at most one solution. More precisely, there is a positive constant $M_{0}$, depending only on the quantities in assumptions (2.1), (2.2), (2.3), (2.4), (2.5), and (2.9), and the length $T$ of the time interval, such that

$$
\begin{aligned}
& \left|u_{1}(t)-u_{2}(t)\right|+\left|w_{1}(t)-w_{2}(t)\right| \\
& \quad \leq e^{M_{0}\left(1+\left|u_{01}\right|^{7}+\left|u_{02}\right|^{7}\right)}\left(\left|u_{01}-u_{02}\right|+\left|w_{01}-w_{02}\right|\right) \quad \forall t \in[0, T],
\end{aligned}
$$

for any two solutions $\left\{u_{i}, w_{i}\right\}$ of (1.1), (1.2) with initial values $u_{0 i}, w_{0 i}, i=1,2$.

Remark 2.5. We see from the proof of Theorem 2.4 given in Section 4 that the constant $M_{0}$ is of the form $e^{K_{1}(1+T)}$ for a positive constant depending only on the quantities in assumptions (2.1), (2.2), (2.3), (2.4), (2.5), and (2.9).

Remark 2.6. As is easily understood, for the construction of a local in time solution of (1.1), (1.2), and (1.3) it is enough to assume that the functions $a_{i}, b_{i}, g$, $h, f_{*}, f^{*}$ are locally Lipschitz continuous and the inequalities in (2.4) and (2.7) are satisfied as well in a neighborhood of the initial point $\left(u_{0}, w_{0}\right)$.

As is easily checked, without loss of generality, we may assume that the functions $a_{i}, b_{i}, i=1,2, g$, and $h$ are globally Lipschitz continuous on $\mathbb{R}^{2}$ and all the inequalities in (2.4) hold on $\mathbb{R}^{2}$. In fact, it is enough to extend them outside $\mathscr{F}$, 
for instance, in the following manner:

$$
\begin{array}{lll}
a_{i}(u, w)=a_{i}\left(u, f^{*}(u)\right), & b_{i}(u, w)=b_{i}\left(u, f^{*}(u)\right), & \text { for } w>f^{*}(u), \\
a_{i}(u, w)=a_{i}\left(u, f_{*}(u)\right), & b_{i}(u, w)=b_{i}\left(u, f_{*}(u)\right), & \text { for } w<f_{*}(u), \\
g(u, w)=g\left(u, f^{*}(u)\right), & h(u, w)=h\left(u, f^{*}(u)\right), & \text { for } w>f^{*}(u), \\
g(u, w)=g\left(u, f_{*}(u)\right), & h(u, w)=h\left(u, f_{*}(u)\right), & \text { for } w<f_{*}(u) .
\end{array}
$$

In the rest of this paper, we always assume such extended conditions for functions $a_{i}, b_{i}, i=1,2, g$, and $h$.

The key for the proofs of our theorems is found in the following lemma.

Lemma 2.7. Under conditions (2.1), (2.2), (2.3), (2.4), and (2.5), system (1.1), (1.2) is equivalent to the coupling of (1.1) and the following inclusion:

$$
w^{\prime}(t)+\partial I_{u(t)}(w(t)) \ni \tilde{h}(u(t), w(t)) \quad \text { for a.e. } t \in[0, T]
$$

where

$$
\tilde{h}(u, w):=\frac{a_{1}(u, w) h(u, w)-b_{1}(u, w) g(u, w)}{a_{1}(u, w) b_{2}(u, w)-a_{2}(u, w) b_{1}(u, w)}
$$

Proof. In order to eliminate the term $u^{\prime}$ from (1.2), compute $\left((1.2) \times a_{1}-(1.1) \times\right.$ $\left.b_{1}\right) /\left(a_{1} b_{2}-a_{2} b_{1}\right)$. Then we have

$$
w^{\prime}+\frac{a_{1}}{a_{1} b_{2}-a_{2} b_{1}} \partial I_{u}(w) \ni \tilde{h} \quad \text { a.e. on }[0, T]
$$

where $\tilde{h}$ is the same as given by (2.13). Here we note the invariance of $\partial I_{u}(w)$ under multiplication by positive numbers, namely $\partial I_{u}(w)=k \partial I_{u}(w)$ for every positive $k$. In fact, this property is immediately seen from (1.4). Therefore, by (2.4), we have $\left(a_{1} /\left(a_{1} b_{2}-a_{2} b_{1}\right)\right) \partial I_{u}(w)=\partial I_{u}(w)$. Hence (2.12) is obtained.

This lemma shows that it is enough to prove Theorems 2.3 and 2.4 to the system $\{(1.1),(2.12)\}$ instead of $\{(1.1),(1.2)\}$.

\section{A priori bounds of solutions}

In this section, we give a priori bounds of the form (2.8) for solutions under the same assumptions as Theorem 2.3. For the sake of simplicity of notation we denote by $L_{0}$, chosen so that $L_{0}>1$, a common Lipschitz constant of functions $a_{i}, b_{i}, i=1,2, g, h$ on $\mathbb{R}^{2}$; note the extended condition (2.11).

Let $\{u, w\}$ be any solution of (1.1), (1.2) with given initial data $u_{0}, w_{0}$; of course, the relation $f_{*}\left(u_{0}\right) \leq w_{0} \leq f^{*}\left(u_{0}\right)$ is satisfied. Then we prove the following lemma. 
Lemma 3.1. Put $N_{0}:=k_{0}+\left|u_{0}\right|$, where $k_{0}$ is the same number as in (2.1). Then,

$$
\begin{aligned}
-N_{0}-\frac{\left|g\left(-N_{0}, f_{*}\left(-N_{0}\right)\right)\right|}{2 L_{0}^{2}} \exp \left(\frac{2 L_{0}^{2}}{\delta_{0}} T\right) \\
\quad \leq u(t) \leq N_{0}+\frac{\left|g\left(N_{0}, f^{*}\left(N_{0}\right)\right)\right|}{2 L_{0}^{2}} \exp \left(\frac{2 L_{0}^{2}}{\delta_{0}} T\right)
\end{aligned}
$$

for all $t \in[0, T]$.

Proof. We multiply $(1.1)$ by $\left(u-N_{0}\right)^{+}$to get

$$
a_{1} u^{\prime}\left(u-N_{0}\right)^{+}+a_{2} w^{\prime}\left(u-N_{0}\right)^{+}=g\left(u-N_{0}\right)^{+} .
$$

Since $w=f^{*}(u)$ for $u \geq N_{0}$ by (2.1), it follows from the above equality that

$$
\frac{1}{2}\left(a_{1}+a_{2} f^{*^{\prime}}(u)\right) \frac{d}{d t}\left|\left(u-N_{0}\right)^{+}\right|^{2}=g\left(u-N_{0}\right)^{+}
$$

Also, we note that

$$
\begin{aligned}
g\left(u-N_{0}\right)^{+}= & \left(g\left(u, f^{*}(u)\right)-g\left(N_{0}, f^{*}\left(N_{0}\right)\right)\right)\left(u-N_{0}\right)^{+} \\
& +g\left(N_{0}, f^{*}\left(N_{0}\right)\right)\left(u-N_{0}\right)^{+} \\
\leq & 2 L_{0}^{2}\left|\left(u-N_{0}\right)^{+}\right|^{2}+\left|g\left(N_{0}, f^{*}\left(N_{0}\right)\right)\right|\left(u-N_{0}\right)^{+} .
\end{aligned}
$$

By (3.3) and (3.4) with assumption (2.7) we have

$$
\frac{d}{d t}\left(u-N_{0}\right)^{+} \leq \frac{2 L_{0}^{2}}{\delta_{0}}\left(u-N_{0}\right)^{+}+\frac{1}{\delta_{0}}\left|g\left(N_{0}, f^{*}\left(N_{0}\right)\right)\right|
$$

and the second inequality of (3.1) is obtained. The first inequality is similarly obtained, too.

COROLlary 3.2. There is a positive constant $K_{0}^{(1)}$, depending only on the quantities in (2.1), (2.2), (2.3), (2.4), (2.5), (2.6), and (2.7), such that

$$
|u(t)|+|w(t)| \leq e^{K_{0}^{(1)}(1+T)}\left(1+\left|u_{0}\right|\right), \quad 0 \leq \forall t \leq T
$$

Proof. We note that $|w|$ is bounded by a linear function of $|u|$ and hence so are $\left|g\left(N_{0}, f^{*}\left(N_{0}\right)\right)\right|$ and $\left|g\left(-N_{0}, f_{*}\left(-N_{0}\right)\right)\right|$. Therefore, from (3.1) together with this fact we immediately derive (3.6) for a certain positive constant $K_{0}^{(1)}$. 
Lemma 3.3. There is a positive constant $K_{0}^{(2)}$, depending only on the quantities in (2.1), (2.2), (2.3), (2.4), (2.5), (2.6), and (2.7), such that

$$
\left|u^{\prime}(t)\right|+\left|w^{\prime}(t)\right| \leq e^{K_{0}^{(2)}(1+T)}\left(1+\left|u_{0}\right|^{3}\right) \quad \text { for a.e. } t \in[0, T]
$$

Proof. We have $a_{1}\left|u^{\prime}\right|^{2}+a_{2} w^{\prime} u^{\prime}=g u^{\prime}$ by multiplying (1.1) by $u^{\prime}$. Here, since $u$, $w$ satisfy (2.12), we observe that

$$
w^{\prime}= \begin{cases}\tilde{h} & \text { a.e. on }\left\{t ; f_{*}(u)<w<f^{*}(u)\right\} \\ f_{*}^{\prime}(u) u^{\prime} & \text { a.e. on }\left\{t ; w=f_{*}(u)\right\} \\ f^{*^{\prime}}(u) u^{\prime} & \text { a.e. on }\left\{t ; w=f^{*}(u)\right\}\end{cases}
$$

so that

$$
\begin{aligned}
|g|\left|u^{\prime}\right| \geq & a_{1}\left|u^{\prime}\right|^{2}+a_{2} w^{\prime} u^{\prime} \\
& = \begin{cases}a_{1}\left|u^{\prime}\right|^{2}+a_{2} \tilde{h} u^{\prime} & \text { a.e. on }\left\{t ; f_{*}(u)<w<f^{*}(u)\right\}, \\
\left(a_{1}+a_{2} f_{*}^{\prime}(u)\right)\left|u^{\prime}\right|^{2} & \text { a.e. on }\left\{t ; w=f_{*}(u)\right\}, \\
\left(a_{1}+a_{2} f^{*}(u)\right)\left|u^{\prime}\right|^{2} & \text { a.e. on }\left\{t ; w=f^{*}(u)\right\} .\end{cases}
\end{aligned}
$$

Accordingly, using our assumptions (2.4) and (2.7), we see from the above equality that

$$
\left|u^{\prime}\right|^{2} \leq \begin{cases}\frac{2}{c_{0}^{2}}\left(\left|a_{2} \tilde{h}\right|^{2}+|g|^{2}\right) & \text { a.e. on }\left\{t ; f_{*}(u)<w<f^{*}(u)\right\} \\ \frac{|g|^{2}}{\delta_{0}^{2}} & \text { a.e. on }\left\{t ; w=f_{*}(u) \text { or } f^{*}(u)\right\}\end{cases}
$$

Moreover, note that $|\tilde{h}| \leq \operatorname{const}\left(1+|u|^{2}\right)$ and hence $\left|a_{2} \tilde{h}\right| \leq \operatorname{const}\left(1+|u|^{3}\right)$. Therefore, it follows from (3.10) with (3.6) and (3.8) that an estimate of the form (2.8) holds for a certain positive constant $K_{0}^{(2)}$.

Now, putting $K_{0}:=\max \left\{K_{0}^{(1)}, K_{0}^{(2)}\right\}$, we see the estimate (2.8) for all solutions $\{u, w\}$ with initial data $u_{0}, w_{0}$.

\section{Proof of uniqueness}

In this section, we prove (2.10) for two solutions $\left\{u_{k}, w_{k}\right\}, k=1,2$, of system (1.1), (1.2), and (1.3) for given initial data $u_{0 k}, w_{0 k}$, assuming always that conditions (2.1), (2.2), (2.3), (2.4), (2.5), and (2.9) are satisfied. 
For simplicity we put

$$
\begin{gathered}
a_{i}^{(k)}:=a_{i}\left(u_{k}, w_{k}\right), \quad b_{i}^{(k)}:=b_{i}\left(u_{k}, w_{k}\right), \quad g^{(k)}:=g\left(u_{k}, w_{k}\right), \\
h^{(k)}:=h\left(u_{k}, w_{k}\right), \quad \tilde{h}^{(k)}:=\tilde{h}\left(u_{k}, w_{k}\right), \quad i, k=1,2, \\
\bar{u}:=u_{1}-u_{2}, \quad \bar{w}:=w_{1}-w_{2}, \\
\bar{u}_{0}:=u_{01}-u_{02}, \quad \bar{w}_{0}:=w_{01}-w_{02} .
\end{gathered}
$$

With these notations, by taking the difference of two solutions, we have

$$
a_{1}^{(1)} \bar{u}^{\prime}+a_{2}^{(1)} \bar{w}^{\prime}=\left(g^{(1)}-g^{(2)}\right)-\left(a_{1}^{(1)}-a_{1}^{(2)}\right) u_{2}^{\prime}-\left(a_{2}^{(1)}-a_{2}^{(2)}\right) w_{2}^{\prime} .
$$

Now, take any measurable function $s_{\bar{u}}$ in time so that

$$
s_{\bar{u}} \in \operatorname{sign}(\bar{u})= \begin{cases}1 & \text { if } \bar{u}>0 \\ {[-1,1]} & \text { if } \bar{u}=0 \\ -1 & \text { if } \bar{u}<0\end{cases}
$$

and multiply (4.2) by $s_{\bar{u}}$ and use the Lipschitz continuity of functions $a_{i}, g$. Then, since $\bar{u}^{\prime} s_{\bar{u}}=(d / d t)|\bar{u}|$, we have

$$
a_{1}^{(1)} \frac{d}{d t}|\bar{u}|+a_{2}^{(1)} \bar{w}^{\prime} s_{\bar{u}} \leq L_{0}\left(1+\left|u_{2}^{\prime}\right|+\left|w_{2}^{\prime}\right|\right)(|\bar{u}|+|\bar{w}|)
$$

We arrange this inequality in the following form:

$$
\frac{d}{d t}\left\{a_{1}^{(1)}|\bar{u}|\right\}+a_{2}^{(1)} \bar{w}^{\prime} s_{\bar{u}} \leq L_{0}\left(1+\left|u_{1}^{\prime}\right|+\left|u_{2}^{\prime}\right|+\left|w_{1}^{\prime}\right|+\left|w_{2}^{\prime}\right|\right)(|\bar{u}|+|\bar{w}|) .
$$

Next, we show that

$$
\bar{w}^{\prime} s_{\bar{u}} \geq \frac{d}{d t}|\bar{w}|-2 L_{1}(|\bar{u}|+|\bar{w}|) \quad \text { a.e. on }[0, T]
$$

where $L_{1}$ is a Lipschitz constant of $\tilde{h}$ on $\mathbb{R}^{2}$; note that $L_{1}$ is dominated by a positive number of the form const $\left(1+\left|u_{01}\right|^{3}+\left|u_{02}\right|^{3}\right)$. We show (4.6) on the following four subsets of time $t \in[0, T]:$ (a) $E_{1}:=\{t ; \bar{u} \geq 0, \bar{w}<0\}$, (b) $E_{2}:=$ $\{t ; \bar{u} \leq 0, \bar{w}>0\}$, (c) $E_{3}:=\{t ; \bar{u} \geq 0, \bar{w} \geq 0\}$, (d) $E_{4}:=\{t ; \bar{u} \leq 0, \bar{w} \leq 0\}$. On the set $E_{3} \cup E_{4}$, by the definition of the function $\operatorname{sign}(\cdot)$, we can choose $s_{\bar{w}}$ so that $s_{\bar{w}}=s_{\bar{u}}$. Therefore, (4.6) trivially holds. Next, we consider the case (a). In this case observe that

$$
w_{1}<f^{*}\left(u_{1}\right), \quad w_{2}>f_{*}\left(u_{2}\right) \quad \text { on } E_{1} \text {. }
$$


In fact, if not, then $w_{1}=f^{*}\left(u_{1}\right) \geq f^{*}\left(u_{2}\right) \geq w_{2}$ or $w_{2}=f_{*}\left(u_{2}\right) \leq f_{*}\left(u_{1}\right) \leq w_{1}$, which contradicts our assumption. By the definition of $\partial I_{u_{1}}\left(w_{1}\right)$ we see that

$$
\left(\tilde{h}^{(1)}-w_{1}^{\prime}\right)\left(z-w_{1}\right) \leq 0, \quad f_{*}\left(u_{1}\right) \leq \forall z \leq f^{*}\left(u_{1}\right) .
$$

Taking $f^{*}\left(u_{1}\right)$ as $z$ in $(4.8)$ yields that $\tilde{h}^{(1)} \leq w_{1}^{\prime}$ on $E_{1}$ and similarly $\tilde{h}^{(2)} \geq w_{2}^{\prime}$ on $E_{1}$. Consequently, it follows that

$$
\bar{w}^{\prime}-\left(\tilde{h}^{(1)}-\tilde{h}^{(2)}\right) \geq 0 \quad \text { on } E_{1} .
$$

Since $s_{\bar{w}}=-1 \leq s_{\bar{u}}$ on $E_{1}$, we derive by multiplying the above inequality by $s_{\bar{u}}-s_{\bar{w}}(\geq 0)$ that

$$
\bar{w}^{\prime} s_{\bar{u}} \geq \frac{d}{d t}|\bar{w}|+\left(\tilde{h}^{(1)}-\tilde{h}^{(2)}\right)\left(s_{\bar{u}}-s_{\bar{w}}\right) \quad \text { a.e. on } E_{1}
$$

This is easily arranged to the form (4.6). Just as (a), we see that inequality (4.6) holds a.e. on $E_{2}$, too. Thus (4.6) holds a.e. on $[0, T]$.

Moreover, multiply (4.6) by $a_{2}^{(1)}\left(\geq c_{0}\right)$ to get

$$
\begin{aligned}
a_{2}^{(1)} \bar{w}^{\prime} s_{\bar{u}} \geq & a_{2}^{(1)} \frac{d}{d t}|\bar{w}|-2 a_{2}^{(1)} L_{1}(|\bar{u}|+|\bar{w}|) \\
\geq & \frac{d}{d t}\left\{a_{2}^{(1)}|\bar{w}|\right\} \\
& -\left(L_{0}+2 a_{2}^{(1)} L_{1}\right)\left(1+\left|u_{1}^{\prime}\right|+\left|w_{1}^{\prime}\right|\right)(|\bar{u}|+|\bar{w}|) \quad \text { a.e. on }[0, T] .
\end{aligned}
$$

We then obtain from (4.5) with the above inequality that

$$
\begin{aligned}
& \frac{d}{d t}\left\{a_{1}^{(1)}|\bar{u}|+a_{2}^{(1)}|\bar{w}|\right\} \\
& \leq 2\left(L_{0}+a_{2}^{(1)} L_{1}\right)\left(1+\left|u_{1}^{\prime}\right|+\left|u_{2}^{\prime}\right|+\left|w_{1}^{\prime}\right|+\left|w_{2}^{\prime}\right|\right)(|\bar{u}|+|\bar{w}|) \\
& \leq \frac{2\left(L_{0}+a_{2}^{(1)} L_{1}\right)}{c_{0}}\left(1+\left|u_{1}^{\prime}\right|+\left|u_{2}^{\prime}\right|\right. \\
& \left.\quad+\left|w_{1}^{\prime}\right|+\left|w_{2}^{\prime}\right|\right)\left(a_{1}^{(1)}|\bar{u}|+a_{2}^{(1)}|\bar{w}|\right) \quad \text { a.e. on }[0, T] .
\end{aligned}
$$

Here, on account of estimates (2.8) with the fact that $a_{2}^{(1)} L_{1}$ is dominated by a positive number of the form const $\left(1+\left|u_{01}\right|^{4}+\left|u_{02}\right|^{4}\right)$, the last inequality implies that

$$
\begin{aligned}
& \frac{d}{d t}\left\{a_{1}^{(1)}|\bar{u}|+a_{2}^{(1)}|\bar{w}|\right\} \\
& \quad \leq e^{K_{1}^{\prime}(1+T)}\left(1+\left|u_{01}\right|^{7}+\left|u_{02}\right|^{7}\right)\left(a_{1}^{(1)}|\bar{u}|+a_{2}^{(1)}|\bar{w}|\right) \quad \text { a.e. on }[0, T]
\end{aligned}
$$


where $K_{1}^{\prime}$ is a positive constant depending only on the quantities in assumptions (2.1), (2.2), (2.3), (2.4), (2.5), and (2.9). Therefore,

$$
\begin{aligned}
a_{1}^{(1)}(t) \mid & \bar{u}(t)\left|+a_{2}^{(1)}(t)\right| \bar{w}(t) \mid \\
\leq & \exp \left\{e^{K_{1}^{\prime}(1+T)}\left(1+\left|u_{01}\right|^{7}+\left|u_{02}\right|^{7}\right) T\right\} \\
& \cdot\left(a_{1}^{(1)}(0)\left|\bar{u}_{0}\right|+a_{2}^{(1)}(0)\left|\bar{w}_{0}\right|\right) \quad \forall t \in[0, T] .
\end{aligned}
$$

Now, noting that $a_{1}^{(1)}(0)$ and $a_{2}^{(1)}(0)$ are dominated by a number of the form const $\left(1+\left|u_{01}\right|\right)$, from (4.14) we infer the required inequality (2.10) for a positive number $M_{0}$ of the form $e^{K_{1}(1+T)}$, where $K_{1}$ is a positive constant depending only on the quantities in (2.1), (2.2), (2.3), (2.4), (2.5), and (2.9).

\section{Proof of existence}

Throughout this section assume that the extended assumptions (2.1), (2.2), (2.3), (2.4), (2.5), (2.6), and (2.7) hold and (2.12) as well. Let $u_{0}, w_{0}$ be a pair of initial data such that $\left(u_{0}, w_{0}\right) \in \mathscr{F}$. According to the a priori estimates $(2.8)$ for solutions, we may assume, without loss of generality, that the functions $a_{i}, b_{i}$, $i=1,2, g$ and $\tilde{h}$ are bounded on $\mathbb{R}^{2}$.

Consider approximate problems $\left(P_{\lambda}\right)$, which consist of (5.1), (5.2), and (5.3), with parameter $\lambda \in\left(0, \lambda_{0}\right]$ for a positive number $\lambda_{0}$ small enough, to find a pair of functions $\{u, w\}$ satisfying

$$
\begin{gathered}
a_{1}(u, w) u^{\prime}+a_{2}(u, w) \frac{d}{d t} J(u, w)=g(u, w) \quad \text { a.e. on }[0, T] \\
w^{\prime}+\partial I_{u}^{\lambda}(w)=\tilde{h}(u, w) \quad \text { a.e. on }[0, T]
\end{gathered}
$$

subject to the initial conditions

$$
u(0)=u_{0}, \quad w(0)=w_{0}
$$

where $J(u, \cdot)$ is the projection mapping from $\mathbb{R}$ onto $\left[f_{*}(u), f^{*}(u)\right]$, namely

$$
J(u, w)=w-\left(w-f^{*}(u)\right)^{+}+\left(f_{*}(u)-w\right)^{+} \quad \forall w \in \mathbb{R}
$$

and $\partial I_{u}^{\lambda}$ is the Yosida approximation of $\partial I_{u}$, namely

$$
\partial I_{u}^{\lambda}(w)=\frac{\left(w-f^{*}(u)\right)^{+}}{\lambda}-\frac{\left(f_{*}(u)-w\right)^{+}}{\lambda} \quad \forall w \in \mathbb{R}
$$


Clearly, $J$ is Lipschitz continuous on $\mathbb{R}^{2}$ and nondecreasing in both variables, and $\partial I_{u}^{\lambda}$ is Lipschitz continuous on $\mathbb{R}^{2}$ and nondecreasing in $w$. Also, $\partial I_{u}^{\lambda}(\cdot)$ is the subdifferential of the convex function

$$
I_{u}^{\lambda}(w):=\frac{\left|\left(w-f^{*}(u)\right)^{+}\right|^{2}}{2 \lambda}+\frac{\left|\left(f_{*}(u)-w\right)^{+}\right|^{2}}{2 \lambda} \quad \forall w \in \mathbb{R} .
$$

Lemma 5.1. For each parameter $\lambda \in\left(0, \lambda_{0}\right]$, problem $\left(P_{\lambda}\right)$ has a solution $\left\{u_{\lambda}, w_{\lambda}\right\}$ such that $u_{\lambda}$ and $w_{\lambda}$ are Lipschitz continuous on $[0, T]$. Moreover, the following uniform estimates hold: there is a positive constant $R_{1}$ independent of $\lambda \in\left(0, \lambda_{0}\right]$ such that

$$
\begin{gathered}
\left|u_{\lambda}(t)\right|+\left|w_{\lambda}(t)\right| \leq R_{1} \quad \forall t \in[0, T], \\
\left|u_{\lambda}^{\prime}(t)\right| \leq R_{1} \quad \text { for a.e. } t \in[0, T], \quad\left|w_{\lambda}^{\prime}\right|_{L^{2}(0, T)} \leq R_{1} .
\end{gathered}
$$

Proof. Let $J_{\varepsilon}$ be the regularization $J$ by means of the usual mollifier $\rho_{\varepsilon}, 0<\varepsilon \leq \varepsilon_{0}$ ( $\varepsilon_{0}$ is a positive number close enough to 0 ), namely

$$
J_{\varepsilon}(u, w):=\int_{\mathbb{R}^{2}} J(\zeta, \xi) \rho_{\varepsilon}(u-\zeta, w-\xi) d \zeta d \xi \quad \forall(u, w) \in \mathbb{R}^{2} .
$$

Consider the further regularized approximate equation of (5.1)

$$
a_{1}(u, w) u^{\prime}+a_{2}(u, w) \frac{d}{d t} J_{\varepsilon}(u, w)=g(u, w) \quad \text { on }[0, T] .
$$

This is written in the form

$$
\left\{a_{1}(u, w)+a_{2}(u, w) \frac{\partial}{\partial u} J_{\varepsilon}(u, w)\right\} u^{\prime}+a_{2}(u, w) \frac{\partial}{\partial w} J_{\varepsilon}(u, w) w^{\prime}=g(u, w)
$$

Note here that

$$
\frac{\partial}{\partial u} J(u, w)\left(\text { resp., } \frac{\partial}{\partial w} J(u, w)\right)= \begin{cases}f^{*^{\prime}}(u)(\text { resp., } 0) & \text { if } w>f^{*}(u) \\ 0(\text { resp., 1) } & \text { if } f_{*}(u)<w<f^{*}(u) \\ f_{*}^{\prime}(u)(\text { resp., } 0) & \text { if } w<f_{*}(u),\end{cases}
$$

which shows that

$$
0 \leq \frac{\partial}{\partial u} J_{\varepsilon}(u, w) \leq f^{*^{\prime}}(u)\left(\text { resp., } f_{*}^{\prime}(u)\right)+\tau_{\varepsilon}
$$

in the $\varepsilon$-neighborhood of $\left\{(u, w) ; w \geq f^{*}(u)\right.$ (resp., $\left.\left.w \leq f_{*}(u)\right)\right\}$, where

$$
\tau_{\varepsilon}:=\sup \left\{\left|J\left(u_{1}, w_{1}\right)-J\left(u_{2}, w_{2}\right)\right| ;\left(\left|u_{1}-u_{2}\right|^{2}+\left|w_{1}-w_{2}\right|^{2}\right)^{1 / 2} \leq \varepsilon\right\} ;
$$

clearly $\tau_{\varepsilon} \rightarrow 0$ as $\varepsilon \rightarrow 0$. Therefore, on account of (2.4), (2.7), and (2.11), we see that the coefficient $a_{1}(u, w)+a_{2}(u, w)(\partial / \partial u) J_{\varepsilon}(u, w)$ of $u^{\prime}$ in $(5.10)$ is bounded 
from below by a positive constant $\delta_{0}^{\prime}$ on $\mathbb{R}^{2}$, where $\delta_{0}^{\prime}$ is independent of $\lambda \in\left(0, \lambda_{0}\right]$ and $\varepsilon \in\left(0, \varepsilon_{0}\right]$. Therefore, system (5.9) and (5.2) is written in the form

$$
\begin{aligned}
u^{\prime} & =\frac{g(u, w)-a_{2}(u, w)(\partial / \partial w) J_{\varepsilon}(u, w)\left(\tilde{h}(u, w)-\partial I_{u}^{\lambda}(w)\right)}{a_{1}(u, w)+a_{2}(u, w)(\partial / \partial u) J_{\varepsilon}(u, w)}, \\
w^{\prime} & =\tilde{h}(u, w)-\partial I_{u}^{\lambda}(w)
\end{aligned}
$$

and the right-hand sides are Lipschitz continuous in $(u, w)$. By the general existence-uniqueness theorem for ODEs, problem (5.9) and (5.2) with initial condition (5.3) admits a unique solution, denoted by $\left\{u_{\lambda \varepsilon}, w_{\lambda_{\varepsilon}}\right\}$.

Next, we give uniform estimates for $\left\{u_{\lambda_{\varepsilon}}, w_{\lambda_{\varepsilon}}\right\}$ in $\varepsilon$ and $\lambda$. To do so, multiply (5.9) by $\left(u_{\lambda \varepsilon}-N_{0}^{\prime}\right)^{+}$, with $N_{0}^{\prime}:=k_{0}+1+\left|u_{0}\right|$. Then, just as (3.1) of Lemma 3.1, we see that

$$
\left|u_{\lambda \varepsilon}(t)\right| \leq K_{2}, \quad\left|J_{\varepsilon}\left(u_{\lambda \varepsilon}(t), w_{\lambda \varepsilon}(t)\right)\right| \leq K_{2}, \quad 0 \leq \forall t \leq T,
$$

for a positive constant $K_{2}$ independent of $\lambda$ and $\varepsilon$. From these uniform estimates and (5.14) with (5.11) we derive easily that for each $\lambda>0$ small enough, there is a positive constant $K_{3}(\lambda)$ independent of $\varepsilon$ such that

$$
\left|u_{\lambda \varepsilon}^{\prime}\right| \leq K_{3}(\lambda), \quad\left|w_{\lambda \varepsilon}^{\prime}\right| \leq K_{3}(\lambda), \quad\left|\frac{d}{d t} J_{\varepsilon}\left(u_{\lambda \varepsilon}, w_{\lambda \varepsilon}\right)\right| \leq K_{3}(\lambda), \quad \text { a.e. on }[0, T] \text {. }
$$

Therefore, it is possible to extract a null sequence $\left\{\varepsilon_{n}\right\}$ such that $u_{\lambda \varepsilon_{n}}$ and $w_{\lambda \varepsilon_{n}}$ converge to some Lipschitz continuous functions $u_{\lambda}$ and $w_{\lambda}$ uniformly on $[0, T]$, respectively, as $n \rightarrow+\infty$. Moreover, it is easy to see that the pair $\left\{u_{\lambda}, w_{\lambda}\right\}$ is a solution of $\left(P_{\lambda}\right)$; note from $(5.15)$ that

$$
\left|u_{\lambda}(t)\right| \leq K_{2}, \quad 0 \leq \forall t \leq T
$$

Taking account of (5.17) and the expression

$$
\frac{d}{d t} J\left(u_{\lambda}, w_{\lambda}\right)= \begin{cases}\tilde{h}\left(u_{\lambda}, w_{\lambda}\right) & \text { a.e. on }\left\{t ; f_{*}\left(u_{\lambda}\right) \leq w_{\lambda} \leq f^{*}\left(u_{\lambda}\right)\right\} \\ f^{*}\left(u_{\lambda}\right) u_{\lambda}^{\prime} & \text { a.e. on }\left\{t ; w>f^{*}\left(u_{\lambda}\right)\right\} \\ f_{*}^{\prime}\left(u_{\lambda}\right) u_{\lambda}^{\prime} & \text { a.e. on }\left\{t ; w<f^{*}\left(u_{\lambda}\right)\right\}\end{cases}
$$

just as (3.10) in the proof of Lemma 3.3, we obtain by condition (2.7) that

$$
\left|u_{\lambda}^{\prime}\right| \leq K_{4} \quad \text { a.e. on }[0, T]
$$

for a positive constant $K_{4}$ independent of $\lambda>0$.

Finally, multiplying (5.2) by $w_{\lambda}^{\prime}$, we have

$$
\left|w_{\lambda}^{\prime}\right|^{2}+\partial I_{u_{\lambda}}^{\lambda}\left(w_{\lambda}\right) \cdot w_{\lambda}^{\prime}=\tilde{h} w_{\lambda}^{\prime} .
$$


Here, use the following inequality which is derived directly from the expressions (5.4), (5.5), and (5.6):

$$
\frac{d}{d t} I_{u_{\lambda}}^{\lambda}\left(w_{\lambda}\right)-\partial I_{u_{\lambda}}^{\lambda}\left(w_{\lambda}\right) \cdot w_{\lambda}^{\prime} \leq L_{0}\left|u_{\lambda}^{\prime}\right|\left|\partial I_{u_{\lambda}}^{\lambda}\left(w_{\lambda}\right)\right| \quad \text { a.e. on }[0, T]
$$

Then, noting this with (5.19), we immediately obtain

$$
\left|w_{\lambda}^{\prime}\right|^{2}+\frac{d}{d t} I_{u_{\lambda}}^{\lambda}\left(w_{\lambda}\right) \leq L_{0} K_{4}\left(\left|w_{\lambda}^{\prime}\right|+|\tilde{h}|\right) \quad \text { a.e. on }[0, T] \text {. }
$$

From this inequality it is easy to get a uniform estimate for $\left|w_{\lambda}^{\prime}\right|_{L^{2}(0, T)}$ with respect to $\lambda$, since $\tilde{h}:=\tilde{h}\left(u_{\lambda}, w_{\lambda}\right)$ is uniformly bounded and $I_{u_{0}}^{\lambda}\left(w_{0}\right)=0$. Thus uniform estimates of the form (5.7) hold for a positive constant $R_{1}$ independent of $\lambda$.

By virtue of Lemma 5.1 there is a null sequence $\left\{\lambda_{n}\right\}$ such that the solution $u_{n}:=u_{\lambda_{n}}, w_{n}:=w_{\lambda_{n}}$ of $\left(P_{\lambda_{n}}\right)$ constructed above converge to some functions $u, w$ uniformly on $[0, T]$ as $n \rightarrow+\infty$. Simultaneously, since $\partial I_{u_{n}}^{\lambda_{n}}\left(w_{n}\right)$ $\left(=-w_{n}^{\prime}+\tilde{h}\left(u_{n}, w_{n}\right)\right)$ is bounded in $L^{2}(0, T)$, we see from (5.5) that

$$
\begin{aligned}
& \left(w_{n}-f^{*}\left(u_{n}\right)\right)^{+}-\left(f_{*}\left(u_{n}\right)-w_{n}\right)^{+} \\
& \quad=\lambda_{n} \partial I_{u_{n}}^{\lambda_{n}}\left(w_{n}\right) \longrightarrow 0 \quad \text { in } L^{2}(0, T)(\text { as } n \longrightarrow+\infty),
\end{aligned}
$$

so that $f_{*}(u) \leq w \leq f^{*}(u)$ on $[0, T]$ and

$$
\begin{aligned}
& J\left(u_{n}, w_{n}\right) \longrightarrow w \quad \text { uniformly on }[0, T], \\
& u_{n}^{\prime} \longrightarrow u^{\prime} \quad \text { weakly in } L^{2}(0, T)
\end{aligned}
$$

as well as

$$
\frac{d}{d t} J\left(u_{n}, w_{n}\right) \longrightarrow w^{\prime} \quad \text { weakly in } L^{2}(0, T)
$$

Therefore, the limit $\{u, w\}$ gives a solution of the original problem. Thus the existence proof is now complete.

\section{Some numerical simulations}

Another objective of this paper is to verify by some numerical simulations that our system (1.1), (1.2) is useful enough as the mathematical description of various input-output relations $u \rightarrow w$ of hysteresis type appearing in many nonlinear phenomena, and what is the influence of the choice of the coefficients $a_{i}, b_{i}$, $i=1,2$, and of functions $g, h$ on the behaviour of the orbit $(u(t), w(t))$.

In order to catch the main trends of the input-output relation $u \rightarrow w$ we simply take constants as the coefficients $a_{i}(u, w), b_{i}(u, w), i=1,2$, and mostly linear 
functions as $g(u, w), h(u, w)$. The constants are denoted by the same notations $a_{i}, b_{i}$, and they are chosen so as to satisfy that

$$
a_{1}>0, \quad b_{2}>0, \quad a_{1} b_{2}-a_{2} b_{1}>0 .
$$

Also, in our simulation the functions $f_{*}$ and $f^{*}$ are fixed as follows:

$$
\begin{aligned}
f^{*}(u): & = \begin{cases}-1 & \text { if } u<-1.6 \\
5 u^{2}+16 u+11.8 & \text { if }-1.6 \leq u<-1.4 \\
2 u+2 & \text { if }-1.4 \leq u<-0.6 \\
-5 u^{2}-4 u+0.2 & \text { if }-0.6 \leq u<-0.4 \\
1 & \text { if }-0.4 \leq u\end{cases} \\
f_{*}(u): & \begin{cases}-1 & \text { if } u<0.4 \\
5 u^{2}-4 u-0.2 & \text { if } 0.4 \leq u<0.6 \\
2 u-2 & \text { if } 0.6 \leq u<1.4 \\
-5 u^{2}+16 u-11.8 & \text { if } 1.4 \leq u<1.6 \\
1 & \text { if } 1.6 \leq u .\end{cases}
\end{aligned}
$$

As was mentioned above, the following system is considered in this section:

$$
\begin{gathered}
a_{1} u^{\prime}+a_{2} w^{\prime}=g(u, w), \quad 0<t<T, \\
w^{\prime}+\partial I_{u}(w) \ni \tilde{h}(u, w):=\frac{a_{1} g(u, w)-b_{1} h(u, w)}{a_{1} b_{2}-a_{2} b_{1}}, \quad 0<t<T, \\
u(0)=u_{0}, \quad w(0)=w_{0},
\end{gathered}
$$

where $\partial I_{u}(\cdot)$ is the subdifferential of $I_{u}(\cdot)$ associated with functions $f^{*}$ and $f_{*}$ given by (6.2). Note that (6.4) is equivalent to the original form $b_{1} u^{\prime}+b_{2} w^{\prime}+$ $\partial I_{u}(w) \ni h(u, w)$.

Now let $\lambda$ and $\Delta t$ be positive numbers small enough, and put $t^{k}=k \Delta t(k=$ $1,2, \ldots)$. Then the difference scheme for our numerical simulation is of the form

$$
\begin{gathered}
\frac{a_{1}\left(u^{k+1}-u^{k}\right)}{\Delta t}+\frac{a_{2}\left(w^{k+1}-w^{k}\right)}{\Delta t}=g\left(u^{k}, w^{k}\right), \\
\frac{w^{k+1}-w^{k}}{\Delta t}+\partial I_{u^{k}}^{\lambda}\left(w^{k+1}\right)=\tilde{h}\left(u^{k}, w^{k}\right), \quad k=0,1,2, \ldots, \\
u^{0}=u_{0}, \quad w^{0}=w_{0},
\end{gathered}
$$

where

$$
\partial I_{u^{k}}^{\lambda}\left(w^{k+1}\right)=\frac{\left[w^{k+1}-f^{*}\left(u^{k}\right)\right]^{+}}{\lambda}-\frac{\left[f_{*}\left(u^{k}\right)-w^{k+1}\right]^{+}}{\lambda} .
$$

The graphs of $I_{u}^{\lambda}$ and $\partial I_{u}^{\lambda}$ are illustrated in Figures 6.1 and 6.2, respectively. 


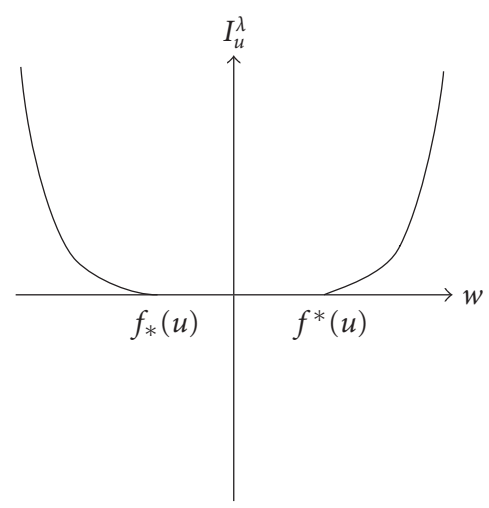

Figure 6.1

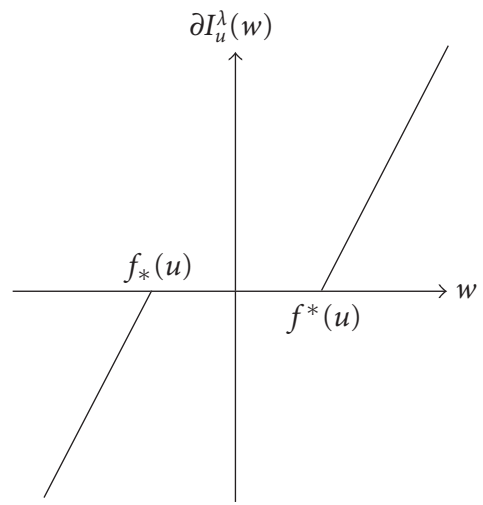

Figure 6.2

In the numerical computations which are performed below, we take

$$
\Delta t=\frac{1}{1000}, \quad \lambda=\frac{1}{10000},
$$

and we examine the following items.

(i) By choice of coefficients $a_{i}, b_{i}$ and functions $g, h$, system (6.3), (6.4) creates various behaviours of the solution pair $(u(t), w(t))$. In particular, it is possible to control by them the trend of clockwise or anti-clockwise behaviour of $(u(t), w(t))$.

(ii) Fixing the coefficients $a_{i}, b_{i}$ and the initial data, we investigate the influence of the functions $g, h$ on the behaviour of $(u(t), w(t))$.

(iii) We investigate the asymptotic behaviour of $(u(t), w(t))$ as $t \rightarrow+\infty$ for various initial data when any other data are fixed. 
578 Systems with hysteresis effect

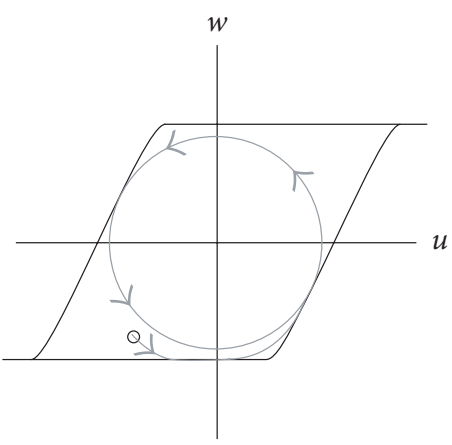

Figure 6.3

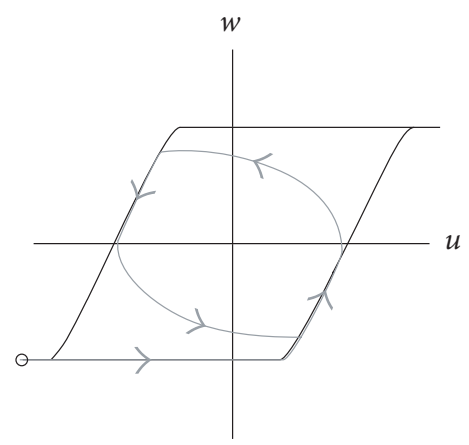

Figure 6.5

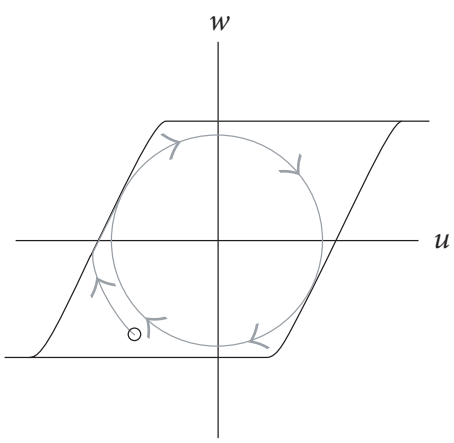

Figure 6.4

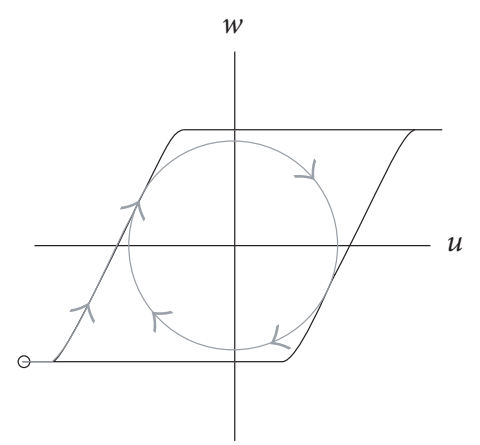

Figure 6.6

Simulation 1. These experiments show that the clockwise and anti-clockwise behaviour of the orbit $(u(t), w(t))$ is created by choosing suitable coefficients for given initial data. It seems that the orbit is periodic in time after a certain time.

\begin{tabular}{lccccccc}
\hline Data table & $a_{1}$ & $a_{2}$ & $b_{1}$ & $b_{2}$ & $h(u, w)$ & $g(u, w)$ & $u_{0}, w_{0}$ \\
\hline Figure 6.3 & 1 & 1 & -1 & 1 & $u+w$ & $u-w$ & $u_{0}=-0.7, w_{0}=-0.8$ \\
Figure 6.4 & 1 & 1 & -1 & 1 & $-u-w$ & $-u+w$ & $u_{0}=-0.7, w_{0}=-0.8$ \\
Figure 6.5 & 1 & 1 & -1 & 1 & $u+3 w$ & $u-2 w$ & $u_{0}=-1.8, w_{0}=-1.0$ \\
Figure 6.6 & 1 & 1 & -3 & 1 & $-u-w$ & $-u+w$ & $u_{0}=-1.8, w_{0}=-1.0$ \\
\hline
\end{tabular}




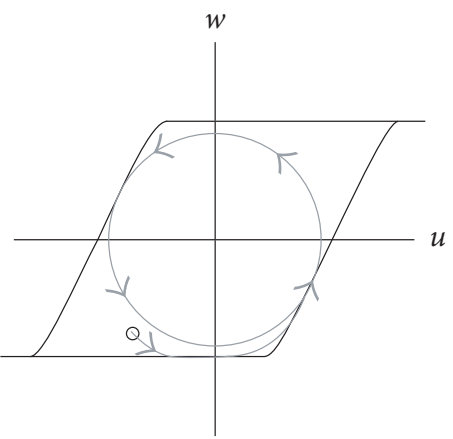

Figure 6.7

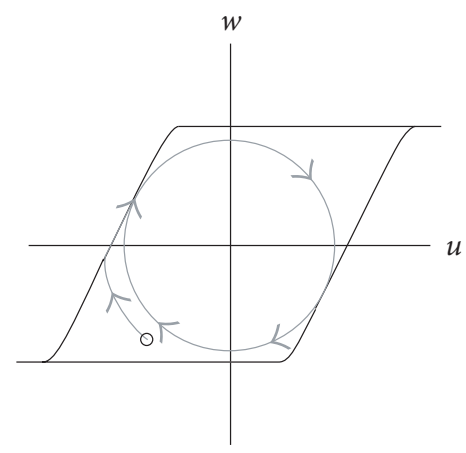

Figure 6.9

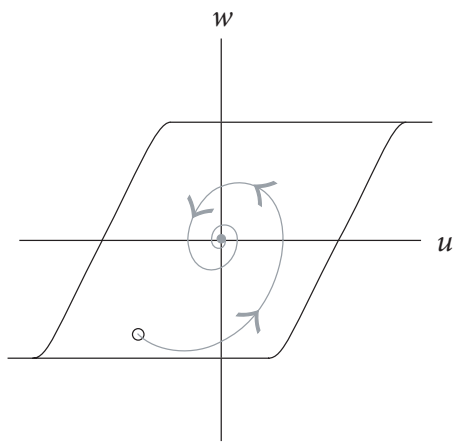

Figure 6.8

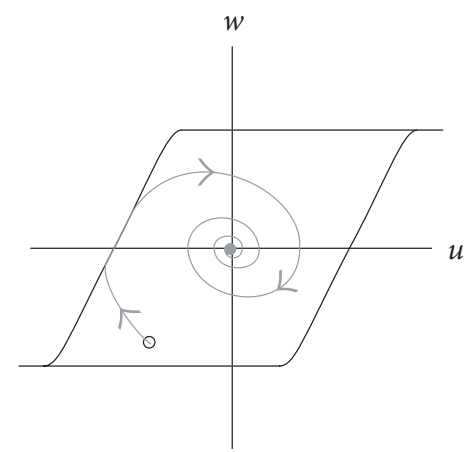

Figure 6.10

Simulation 2. These experiments show that $b_{1}=-1$ gives an anti-clockwise (resp., a clockwise) periodic behaviour of the orbit $(u(t), w(t))$ in time. When the value of $b_{1}$ becomes smaller (resp., larger), the orbit asymptotically converges anti-clockwise (resp., clockwise) to a stable point along a spiral curve.

\begin{tabular}{lccccccc}
\hline Data table & $a_{1}$ & $a_{2}$ & $b_{1}$ & $b_{2}$ & $h(u, w)$ & $g(u, w)$ & $u_{0}, w_{0}$ \\
\hline Figure 6.7 & 1 & 1 & -1 & 1 & $u+w$ & $u-w$ & $u_{0}=-0.7, w_{0}=-0.8$ \\
Figure 6.8 & 1 & 1 & -2 & 1 & $u+w$ & $u-w$ & $u_{0}=-0.7, w_{0}=-0.8$ \\
Figure 6.9 & 1 & 1 & -1 & 1 & $-u-w$ & $-u+w$ & $u_{0}=-0.7, w_{0}=-0.8$ \\
Figure 6.10 & 1 & 1 & -0.5 & 1 & $-u-w$ & $-u+w$ & $u_{0}=-0.7, w_{0}=-0.8$ \\
\hline
\end{tabular}




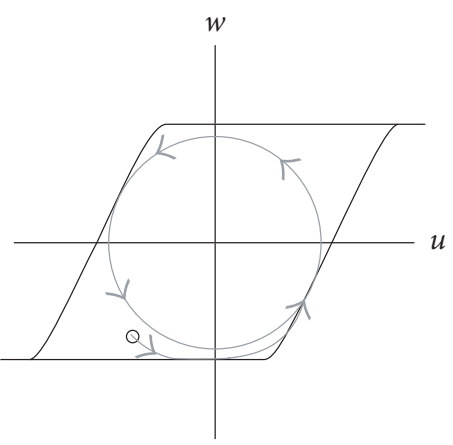

Figure 6.11

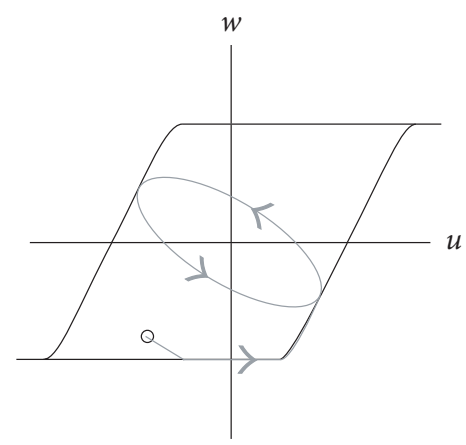

Figure 6.13

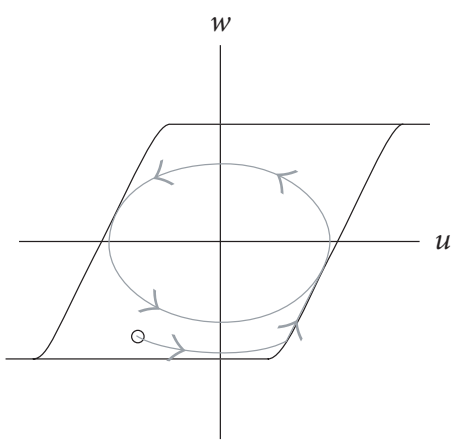

Figure 6.12

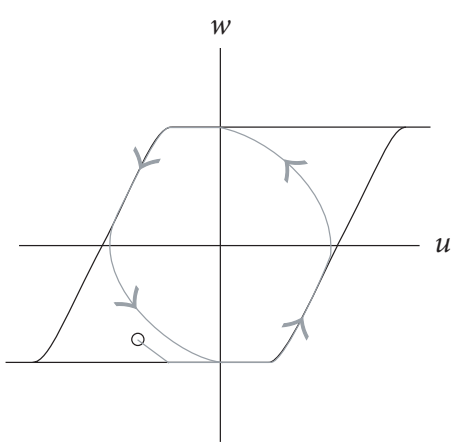

Figure 6.14

Simulation 3. These experiments show that for fixed coefficients $a_{i}, b_{i}, i=1,2$ and initial data as in the table, we can create various (asymptotically) anti-clockwise periodic orbits only by the choice of linear functions $g$, $h$. Also we observe the similar behaviours of orbits in the clockwise case.

\begin{tabular}{cccccccc}
\hline Data table & $a_{1}$ & $a_{2}$ & $b_{1}$ & $b_{2}$ & $h(u, w)$ & $g(u, w)$ & $u_{0}, w_{0}$ \\
\hline Figure 6.11 & 1 & 1 & -1 & 1 & $u+w$ & $u-w$ & $u_{0}=-0.7, w_{0}=-0.8$ \\
Figure 6.12 & 1 & 1 & -1 & 1 & $u+2 w$ & $u-2 w$ & $u_{0}=-0.7, w_{0}=-0.8$ \\
Figure 6.13 & 1 & 1 & -1 & 1 & $2 u+3 w$ & $-w$ & $u_{0}=-0.7, w_{0}=-0.8$ \\
Figure 6.14 & 1 & 1 & -1 & 1 & $u+2 w$ & $u-w$ & $u_{0}=-0.7, w_{0}=-0.8$ \\
\hline
\end{tabular}




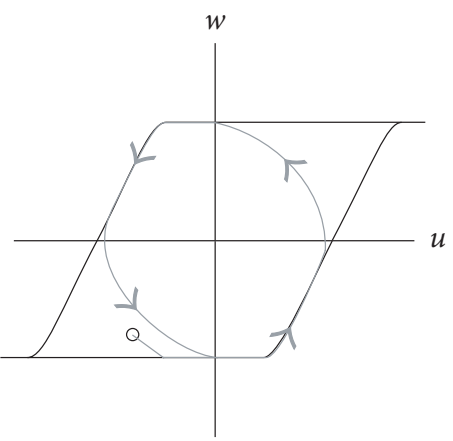

Figure 6.15

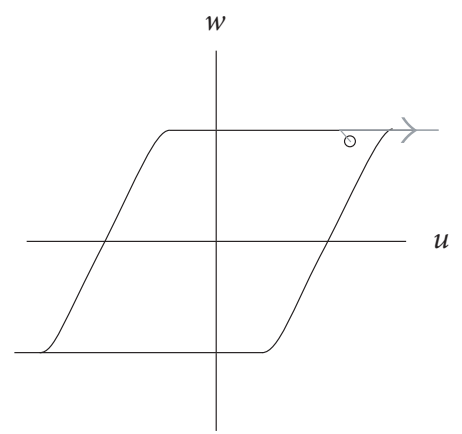

Figure 6.17

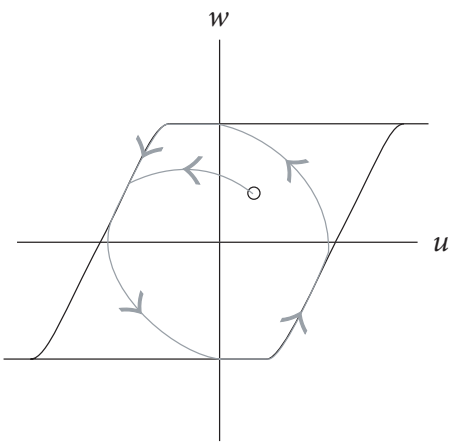

Figure 6.16

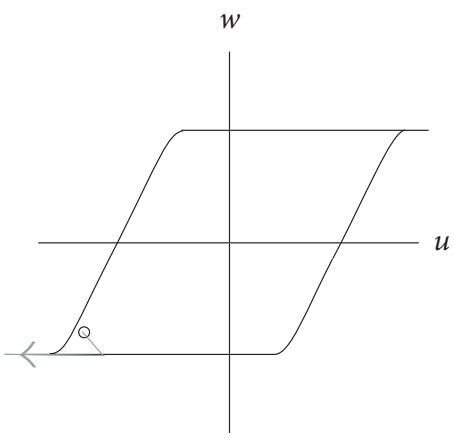

Figure 6.18

Simulation 4. These experiments suggest that the large-time behaviour of orbits $(u(t), w(t))$ is one of the following two cases: (1) the orbit converges to a periodic one as $t \rightarrow+\infty$; (2) the orbit diverges to the point $(-\infty,-1)$ or $(1,+\infty)$ as $t \rightarrow$ $+\infty$.

\begin{tabular}{cccccccc}
\hline Data table & $a_{1}$ & $a_{2}$ & $b_{1}$ & $b_{2}$ & $h(u, w)$ & $g(u, w)$ & $u_{0}, w_{0}$ \\
\hline Figure 6.15 & 1 & 1 & -1 & 1 & $u+2 w$ & $u-w$ & $u_{0}=-0.7, w_{0}=-0.8$ \\
Figure 6.16 & 1 & 1 & -1 & 1 & $u+2 w$ & $u-w$ & $u_{0}=0.4, w_{0}=0.3$ \\
Figure 6.17 & 1 & 1 & -1 & 1 & $u+2 w$ & $u-w$ & $u_{0}=1.2, w_{0}=0.9$ \\
Figure 6.18 & 1 & 1 & -1 & 1 & $u+2 w$ & $u-w$ & $u_{0}=-1.3, w_{0}=-0.8$ \\
\hline
\end{tabular}


582 Systems with hysteresis effect

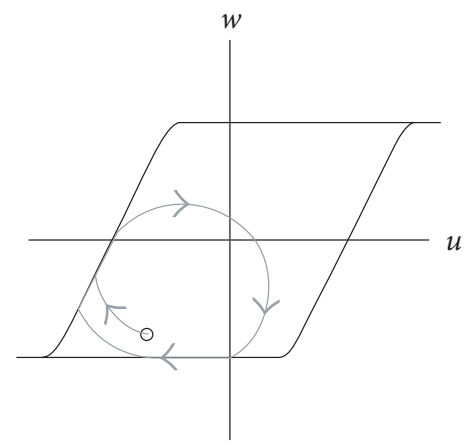

Figure 6.19

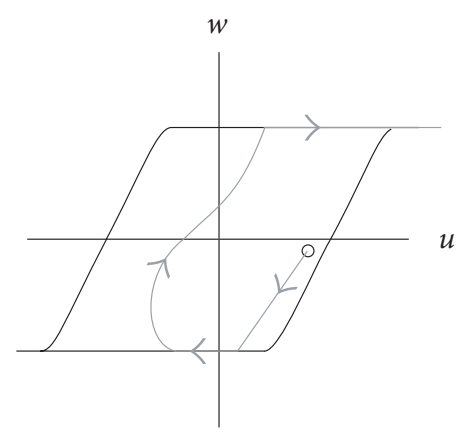

Figure 6.21

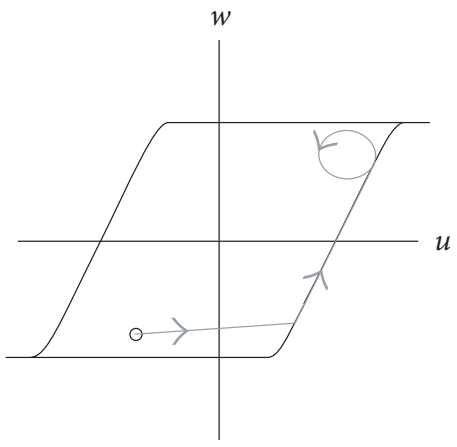

Figure 6.20

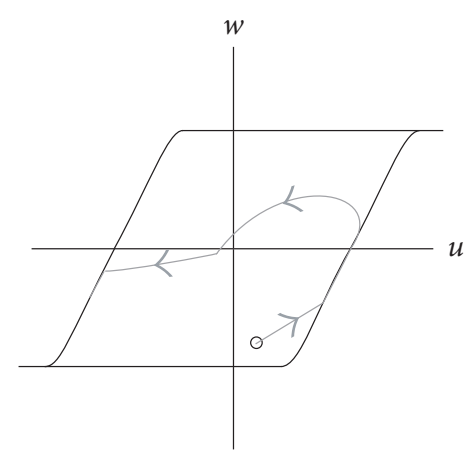

Figure 6.22

Simulation 5 (nonlinear case of $h$ and $g$ ). These experiments show that for fixed coefficients $a_{i}, b_{i}, i=1,2$ as in the table, we can create various behaviours different from the linear case of $h$ and $g$. Figures 6.19 and 6.20 are periodic orbits of $(u(t), w(t))$ in time. Figure 6.21 shows that the orbit diverges to the point $(1,+\infty)$ as $t \rightarrow \infty$. Figure 6.22 shows that the orbit converges to the point $(-1.2,-0.4)$ as $t \rightarrow \infty$.

\begin{tabular}{|c|c|c|c|c|c|c|c|}
\hline Data table & $a_{1}$ & $a_{2}$ & $b_{1}$ & $b_{2}$ & $h(u, w)$ & $g(u, w)$ & $u_{0}, w_{0}$ \\
\hline Figure 6.19 & 1 & 1 & -1 & 1 & $-\sin (u+w)+\cos (u+w)$ & $-u+1.5 w$ & $\begin{aligned} u_{0} & =-0.7 \\
w_{0} & =-0.8\end{aligned}$ \\
\hline Figure 6.20 & 1 & 1 & -1 & 1 & $\sin (u w)-\cos (u w)$ & $u-1.5 w$ & $\begin{aligned} u_{0} & =-0.7 \\
w_{0} & =-0.8\end{aligned}$ \\
\hline Figure 6.21 & 1 & 1 & -1 & 1 & $u w$ & $u w-u+w$ & $\begin{aligned} u_{0} & =0.8 \\
w_{0} & =-0.1\end{aligned}$ \\
\hline Figure 6.22 & 1 & 1 & -1 & 1 & $2 u w$ & $u-3 w$ & $\begin{aligned} u_{0} & =0.2 \\
w_{0} & =-0.8\end{aligned}$ \\
\hline
\end{tabular}




\section{References}

[1] P. Bénilan, Equations d'évolution dans un espace de Banach quelconque et applications, Ph.D. thesis, Univ. Paris-Sud, 1972.

[2] M. Brokate and J. Sprekels, Hysteresis and Phase Transitions, Applied Mathematical Sciences, vol. 121, Springer-Verlag, New York, 1996.

[3] P. Colli, N. Kenmochi, and M. Kubo, A phase-field model with temperature dependent constraint, J. Math. Anal. Appl. 256 (2001), no. 2, 668-685.

[4] M. Hilpert, On uniqueness for evolution problems with hysteresis, Mathematical Models for Phase Change Problems (Óbidos, 1988) (J. F. Rodrigues, ed.), Internat. Ser. Numer. Math., vol. 88, Birkhäuser, Basel, 1989, pp. 377-388.

[5] M. A. Krasnosel'skiǔ and A. V. Pokrovskiŭ, Systems with Hysteresis, Springer-Verlag, Berlin, 1989, translated from the Russian by Marek Niezgódka.

[6] P. Krejčí, Hysteresis operators - a new approach to evolution differential inequalities, Comment. Math. Univ. Carolin. 30 (1989), no. 3, 525-536.

[7] A. Visintin, Differential Models of Hysteresis, Applied Mathematical Sciences, vol. 111, Springer-Verlag, Berlin, 1994.

[8] Models of Phase Transitions, Progress in Nonlinear Differential Equations and Their Applications, vol. 28, Birkhäuser Boston, Massachusetts, 1996.

Emil Minchev and Takanobu Okazaki: Department of Mathematics, Graduate School of Science and Technology, Chiba University, 1-33 Yayoi-chō, Inage-ku, CHIBA, 263-8522, JAPAN

Nobuyuki Kenmochi: Department of Mathematics, Faculty of Education, Chiba University, 1-33 YAYOI-Chō, InAGE-KU, ChibA, 263-8522, JAPAN 


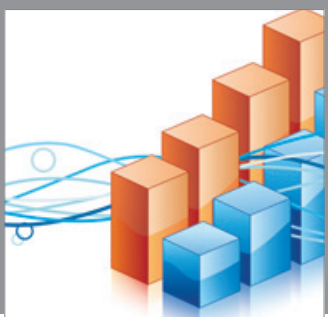

Advances in

Operations Research

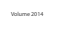

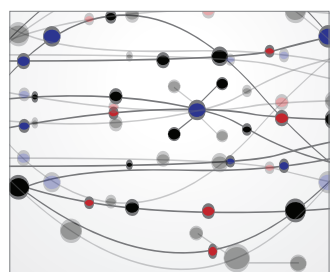

\section{The Scientific} World Journal
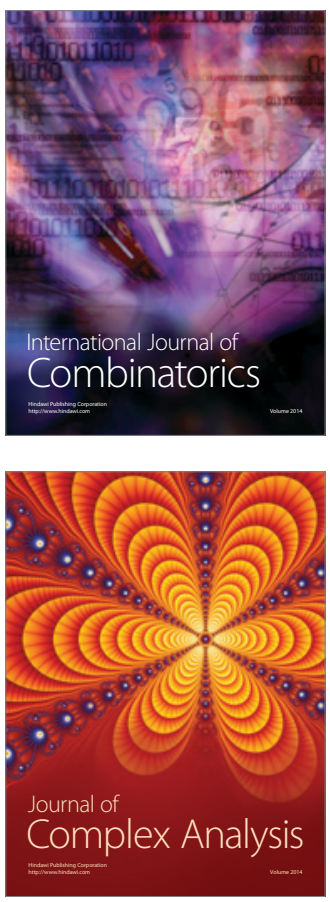

International Journal of

Mathematics and

Mathematical

Sciences
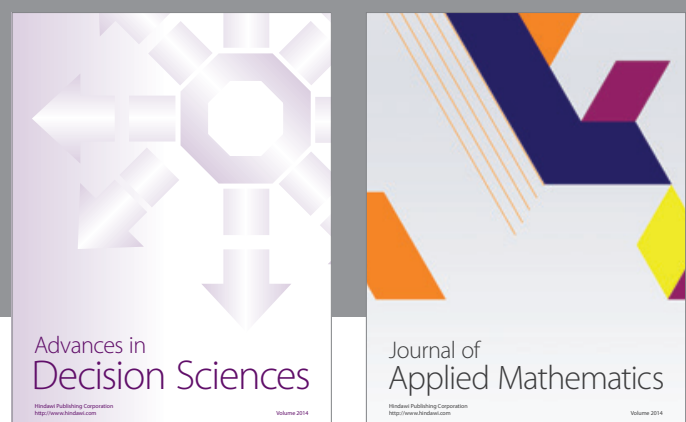

Journal of

Applied Mathematics
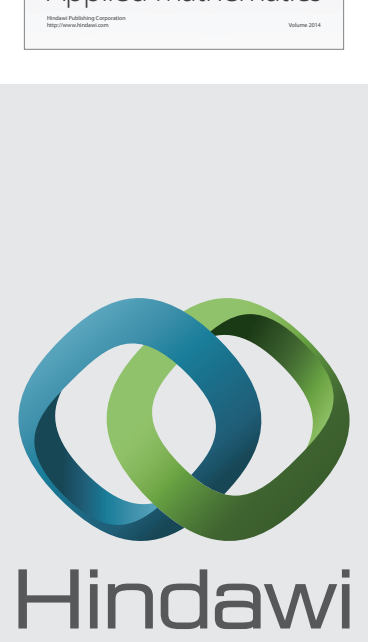

Submit your manuscripts at http://www.hindawi.com
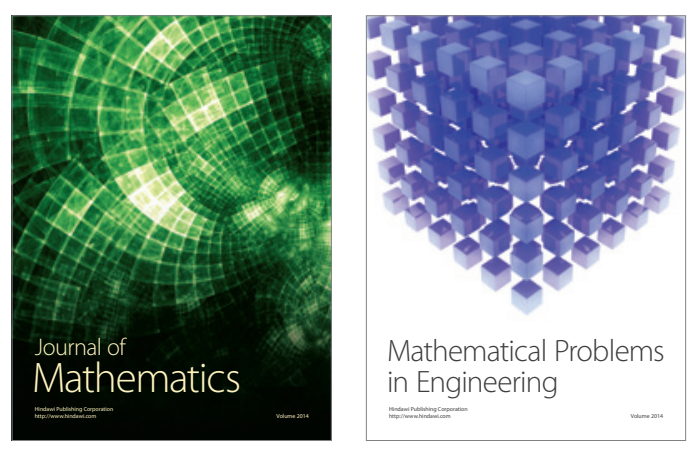

Mathematical Problems in Engineering
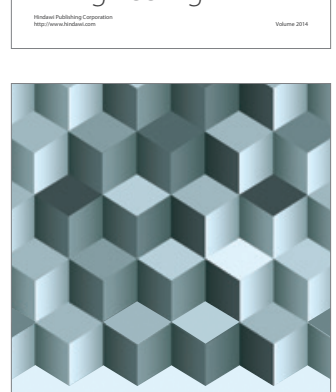

Journal of

Function Spaces
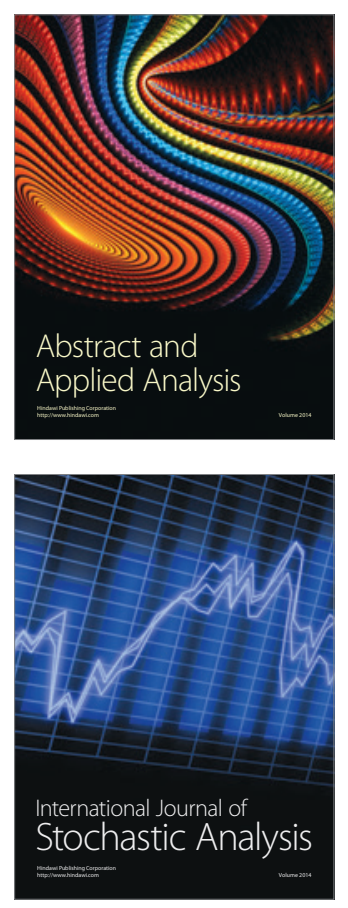

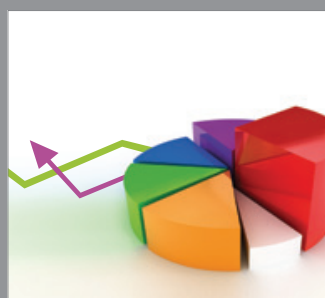

ournal of

Probability and Statistics

Promensencen
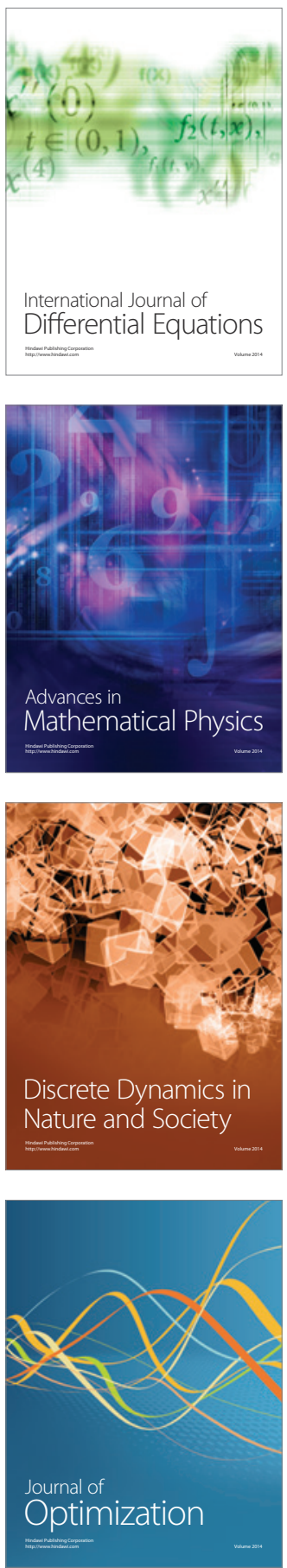